\title{
HOẠT TÍNH SINH HỌC CỦA DOXORUBICIN KHI KẾT HỢP VỚI CAO CHIẾT CỦA NĂM LOÀI HỌ GỬNG (ZINGIBERACEAE)
}

\author{
NGUYẼ̃N THỊ NHẬT THẮNG \\ Khoa Công nghệ hóa học, Truờng Đại học Công nghiệp Thành Phố Hồ Chí Minh \\ nguyenthinhatthang@iuh.edu.vn
}

\begin{abstract}
Tóm tắt. Thân rễ của các loài Zingiber officinale Roscoe (ZO), Zingiber zerumbet (L.) Roscoe ex Sm. (ZZ), Curcuma clovisii Škornič (CC), Curcuma zedoaria (Christm.) Roscoe (CZ) và quả của Amomum villosum Lour $(\mathrm{AV})$ được sử dụng để khảo sát lý trích cao chiết bằng phương pháp ngâm dầm trong ba loại dung môi hữu cơ khác nhau dưới điều kiện tránh sáng tại nhiệt độ phòng. Các dung môi sử dụng bao gồm chloroform $(\mathrm{TCM})$, ethyl aceate $(\mathrm{AcOEt})$ và ethanol $(\mathrm{EtOH})$ với các khoảng thời gian ngâm dầm khảo sát gồm $1,7,14$ và 30 ngày. Trong đó, $\mathrm{TCM}$ và $\mathrm{EtOH}$ được đánh giá là hai dung môi thích hợp cho việc trích ly cao chiết. $Z Z$ cho hiệu suất ly trích cao nhất $(8 \%)$ so với các nguyên liệu còn lại. Hoạt tính kháng tế bào ung thư ruột kết HCT-116 và độc tính trên nguyên bào sợi da người HSF của các cao chiết được đánh giá bằng MTT - test. Kết quả cho thấy tại nồng độ $\mathrm{IC}_{25}$, khi kết hợp các cao chiết này với doxorubicin, đều có ảnh hưởng tích cực trên hoạt tính sinh học. Thành phần hóa học của các cao chiết được phân tích bằng phương pháp sắc ký lỏng phối khổ (LC/MS), sắc ký bản mỏng (TLC) và sắc ký cột.
\end{abstract}

Từ khóa. Zingiber officinale, Zingiber zerumbet, Curcuma zedoaria, Curcuma clovisii, Amomum villosum, doxorubicin, hoạt tính sinh học, hoạt tính kháng ung thư, tách chiết, thực phẩm chức năng.

\section{BIOLOGICAL ACTIVITY OF DOXORUBICIN IN COMBINATION WITH EXTRACTS OF FIVE ZINGIBERACEAE SPECIES}

\begin{abstract}
Rhizomes of Zingiber officinale Roscoe (ZO), Zingiber zerumbet (L.) Roscoe ex Sm. (ZZ), Curcuma clovisii Škornič (CC), Curcuma zedoaria (Christm.) Roscoe, CZ) and fruit of Amomum villosum Lour (AV) were investigated for extraction by immersion with different organic solvents at room temperature and away from direct sunlight. The immersion was made with three different solvents, chloroform (TCM), ethyl acetate (AcOEt), and ethanol (EtOH), for periods of 1, 7, 7, 14, and 30 days. TCM and EtOH were significantly the best extracting solvents. Wild ginger rhizomes provided the highest yield of total extract $(8.0 \%)$. The anticancer activity against HCT-116 cells and the cytotoxicity against the human skin fibroblasts HSF cells in vitro were studied following MTT-test. The result demonstrated that ginger extracts showed a good effect on the biological activity of doxorubicin. The chemical composition of obtained extracts were analyzed by using liquid chromatography/mass spectrometry (LC/MS), thin layer chromatography (TLC), column chromatography on silica gel.
\end{abstract}

Keywords. Zingiber officinale, Zingiber zerumbet, Curcuma zedoaria, Curcuma clovisii, Amomum villosum, doxorubicin, cytotoxic activity, anticancer activity, extraction, food supplement.

\section{GIỚI THIỆU}

Các nghiên cứu trong lĩnh vực ngăn chặn và điều trị ung thư đã và đang phát triển hết sức mạnh mẽ. Điều trị ung thư bằng thuốc (hóa trị) được biết đến từ những năm 1970. Hầu hết các loại thuốc sử dụng trong hóa trị hiện đại gây ra độc tính cao trên cơ thể bệnh nhân sau một thời gian trị liệu. Một giải pháp khắc phục vấn đề này được đưa ra là điều chỉnh chế độ và thời gian trị liệu, cụ thể như dùng thuốc với liều thấp hơn, hóa trị liệu kết hợp và tăng khoảng thời gian giữa các đợt điều trị $[1,2]$. Doxorubicin là một trong những thuốc điều trị ung thư đang được sử dụng phổ biến nhất trên thế giới. Doxorubicin sử dụng trong cả liệu pháp đơn trị và điều trị kết hợp $[2,3]$. Việc sử dụng doxorubicin gây ra nhiều tác dụng phụ như tạo máu không cần thiết, giảm bạch cầu, tăng và rối loạn nhịp tim có thể dẫn đến suy tim sau này, các ảnh hưởng tiêu cực đến hệ tiêu hóa và da [4]. Mặc dù vậy độc tính của doxorubicin và vấn đề kháng thuốc của các tế bào ung thư đối với loại thuốc này vẫn chưa được quan tâm đúng mức. Vấn đề đặt ra trong nghiên cứu này là sử dụng doxorubicin kết hợp cùng các hoạt chất hóa học chiết xuất từ nguyên liệu thiên nhiên nhằm mục đích tăng tính sinh khả dụng đồng thời giúp giảm thiểu độc tính do doxorubicin gây ra trên các tế bào khỏe mạnh. 
Trong một số nghiên cứu trước đây, tác giả cùng nhóm nghiên cứu đã chỉ ra rằng các hợp chất hóa học có chứa nhóm chức chalcone trong phân tử thể hiện hoạt tính kháng ung thư cao và độc tính thấp [5]. Để tiếp tục hướng nghiên cứu này, tác giả chọn một số cây họ gừng làm đối tượng chọn lọc. Họ gừng (Zingiberaceae) là một thực vật thuộc ngành Ngọc lan (Magnoliophyta), lớp Hành (Liliopsida), phân lớp Thài lài (Commelinidae), bộ Gừng (Zingiberales) [6]. Riêng ở Việt Nam, họ Gừng hiện biết có 21 chi với hơn 100 loài. Một số thành phần dược lý chính của họ Gừng được chứng minh như Zingerone có chứa cấu trúc chalcone, gingerol, shogaol, paradol, geraniol, zingiberene... có tác dụng kháng khuẩn, kháng viêm, ức chế co thắt dạ dày, giảm cholesterol trong máu, kháng nấm [7-13]. Đặc biệt phải kể đến hai dược tính quan trọng có khả năng sử dụng trong hóa trị ung thư là khả năng ức chế sự tổng hợp prostaglandin PGE2 [14] và khả năng ức chế hoạt tính men ATPase $[15,16]$. Trong đó PGE2 tham gia thúc đẩy tăng trưởng khối $u$ và di căn $[17,18]$ và $A T P a s e$ tham gia vào việc cung cấp phần lớn năng lượng cho sự tăng trưởng của các tế bào ung thư [19]. Việc nghiên cứu cơ chế và tối ưu hóa tác dụng của chiết xuất từ các cây họ gừng cũng như liệu pháp kết hợp các chiết xuấy này với doxorubicin nhằm ngăn ngừa và ức chế sự phát triển của tế bào ung thư đã và đang được các nhà khoa học trên thế giới chú trọng [20-23].

Tác giả hy vọng các hoạt chất sinh học được chiết xuất từ phần rễ của $\mathrm{ZO}, \mathrm{ZZ}, \mathrm{CC}, \mathrm{CZ}$ và quả của $\mathrm{AV}$ sẽ có tác động tích cực lên hoạt tính kháng ung thư đồng thời giảm thiểu được độc tính của doxorubicin in vitro từ đó cho ra đời một loại thực phẩm chức năng mới có thể sử dụng trong hỗ trợ phòng và điều trị ung thư.

\section{THỬC NGHIẸM}

\subsection{Nguyên liệu}

Căn hành các loài ZO, ZZ, CC, CZ thu hoạch theo thứ tự tại huyện Phú Riềng - tỉnh Tây Ninh, tỉnh Bình Phước, tỉnh Bình Thuận và huyện Gò Công - tỉnh Tiền Giang. Qủa AV - thu hoạch tại tỉnh Ninh Thuận. Tất cả nguyên liệu đều thu hoạch vào vụ mùa hè thu (tháng 7 -10). Hầu hết các nguyên liệu sau khi thu lấy phần rễ ban đầu được làm sạch bằng nước, để ráo nước sau đó được tiếp tục loại bỏ vỏ, cắt lát mỏng thành từng miếng nhỏ đem phơi khô dưới ánh nắng mặt trời. Riêng phần quả của $\mathrm{AV}$ sau khi hái lấy quả chín, để cả vỏ, đãi phơi ngay cho thật khô. Quả AV khô đem bóc bỏ vỏ, lấy khối hạt đem phơi hoặc sấy nhẹ (40 $50{ }^{\circ} \mathrm{C}$ ) đến khô. Nguyên liệu sau đó được đem đi nghiền thành bột và bảo quản nơi khô mát, thoáng gió tránh nóng ẩm.

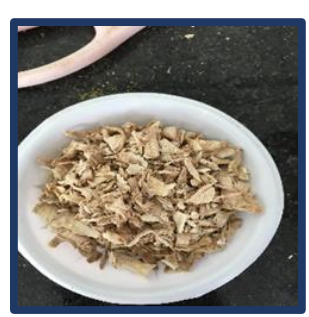

$\mathrm{ZO}$

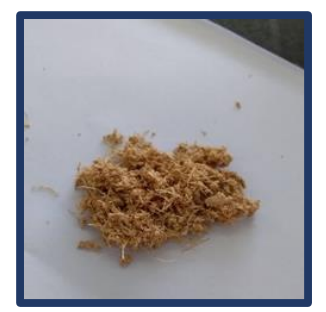

$\mathbf{Z Z}$

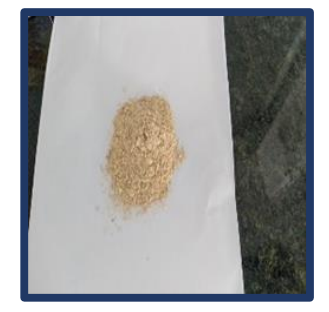

CC

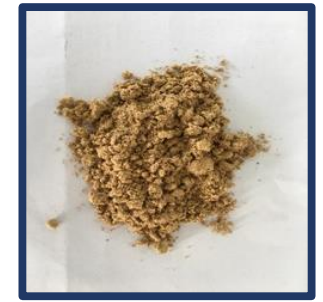

$\mathbf{C Z}$

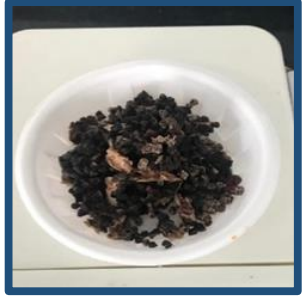

AV

Hình 1. Nguyên liệu sau thu hoạch và xử lý ban đầu

Dung môi sử dụng để khảo sát và chọn lọc điều kiện chiết tối ưu (được mua của hãng Chemsol Việt Nam) gồm Chloroform (99 \%, CAS 67-66-3, TCM), Ethyl acetate (99,5\%, CAS 141-78-6, EtOAc) và Ethanol (99,5\%, CAS 64-17-5, EtOH). Trước khi sử dụng, các tạp chất trong dung môi được loại bỏ bằng phương pháp cô quay chân không (100-200 mbar, $\left.50^{\circ} \mathrm{C}\right)$. Việc thu hồi dung môi để thu cao chiết sử dụng máy cô quay EYELA. Qúa trình định lượng và tính toán hiệu suất tách chiết sử dụng cân điện tử ba số lẻ BAS224S (Sartorius, Đức). Đánh giá kết quả tách chiết bằng phương pháp sắc ký bản mỏng (thin layer chromatography, TLC) sử dụng bản nhôm, silica gel 60 F254 (Merck, Đức), ống mao quản $1 \mu \mathrm{L}$ (Hirchmann, Đức) và đèn UV soi bản mỏng WFH-203 (Trung Quốc, $254 \mathrm{~nm}$ và $365 \mathrm{~nm}$ ). Thành phần cao chiết $\mathrm{ZZ}$ được đánh giá bằng phương pháp sắc ký lỏng ghép khối phổ (liquid chromatography/mass spectrometry, LC/MS) với hệ thống sắc ký Dionex (Mỹ) sử dụng phần mềm Chromeleon phiên bản 7.2.4.8179, hệ thống UV-VIS-3 với bước sóng $280 \mathrm{~nm}$. Đế tách riêng các hoạt chất trong cao chiết tác giả sử dụng phương pháp pháp sắc ký, cột sắc ký Bomex (Trung Quốc, $\emptyset 22 \mathrm{~mm}, h=800 \mathrm{~mm}$ ) có màng lọc và silica gel 60-200 mesh (Merck, Đức).

Quá trình đánh giá hoạt tính sinh học của các cao chiết và doxorubicin sử dụng nguyên bào sợi da người HSF (ATCC PCS-201-012 ${ }^{\mathrm{TM}}$ ) và tế bào ung thư ruột kết người HCT-116 (GSM136316). Các tế bào được 
nuôi cấy trong môi trường D-MEM bổ sung $10 \%$ huyết thanh (PanEko, Russia), L-glutamine, $1 \%$ penicillinstreptomycin trong môi trường vô trùng $5 \% \mathrm{CO}_{2}$ ở $37^{\circ} \mathrm{C}$ cho đến khi hình thành một lớp đơn tế bào trên đáy bình nuôi cấy. Phương pháp MTT-test sử dụng MTT Promega ((3-(4, 5-dimethylthiazol-2-yl)-2, 5diphenyltetrazolium bromide, USA), $\mathrm{DMSO}(\delta \mathrm{H} 2,50, \delta \mathrm{C} 39,52)$ và các đĩa nuôi cấy tế bào gồm 96 giếng nuôi cấy. Mật độ quang của các dung dịch Formazan sau khi ủ tế bào với MTT được đo bằng máy đọc TECAN (Thụy Sĩ).

\subsection{Khảo sát và lựa chọn điều kiện chiết tối ưu}

Các nguyên liệu sau quy trình xử lý ban đầu được đem đi tiến hành khảo sát nhằm tối ưu hóa điều kiện chiết. Cân 10 gram mỗi loại nguyên liệu $\mathrm{ZO}, \mathrm{ZZ}, \mathrm{CC}, \mathrm{CZ}$ và $\mathrm{AV}$. Tiến hành ngâm dầm từng nguyên liệu trong 3 loại dung môi hữu cơ có độ phân cực khác nhau là TCM, AcOEt và EtOH. Thời gian khảo sát ngâm dầm được thực hiện trong khoảng 1 - 30 ngày với 4 mốc khảo sát chính là $1,7,14$ và 30 ngày. Các bình chiết đậy kín, bọc tránh sáng và ngâm dầm ở nhiệt độ phòng. Tại mỗi mốc khảo sát dịch chiết (được kiểm tra TLC) để theo dõi kết quả chiết đạt được. Kết thúc thời gian khảo sát, các mẫu gồm nguyên liệu và dịch chiết được lọc qua giấy lọc (số 101 Advantec, $\mathrm{d}=90$ ) dung dịch sau đó được cô đặc chân không thành dạng cao và tính hiệu suất quá trình chiết.

\subsection{MTT -test}

Cao chiết và doxorubicin được chuẩn bị dưới dạng dung dịch trong dung môi DMSO vô trùng ở nồng độ ban đầu là $1 \mathrm{mg} / \mathrm{mL}$. MTT- test sử dụng các đĩa có 96 giểng nuôi cấy. Các tế bào ( 3 nghìn tế bào/giếng nuôi cấy) được nuôi cấy trong $90 \mu \mathrm{L}$ môi trường $\mathrm{D}-\mathrm{MEM}$ điều kiện tiêu chuẩn $\left(5 \% \mathrm{CO}_{2}, 37^{\circ} \mathrm{C}\right)$ trong 24 giờ để các tể bào bám chặt vào bề mặt của giếng nuôi cấy. Tiếp theo, $10 \mu \mathrm{L}$ dung dịch cao chiết, doxorubicin hoặc hỗn hợp cao chiết-doxorubicin được thêm vào dung dịch nuôi cấy và ủ ở điều kiện tiêu chuẩn trong 72 giờ. Tiếp theo, môi trường với các chế phẩm dược được thay thế bằng $80 \mu \mathrm{L}$ môi trường tinh khiết $\mathrm{D}-$ MEM và $20 \mu \mathrm{L}$ MTT $(5 \mathrm{mg} / \mathrm{mL})$, ủ trong tủ nuôi cấy trong 3 giờ, sau đó môi trường được loại bỏ và thêm $100 \mu \mathrm{L}$ DMSO. Sau 10 phút, mật độ quang của các dung dịch Formazan trong các giếng nuôi cấy đem đi đo ở bước sóng $555 \mathrm{~nm}$ (bước sóng tham chiếu - 650nm) trên máy đọc TECAN (Thụy Sĩ). Kết quả được tính toán và trình bày dưới dạng phần trăm tham chiếu với mẫu trắng (không thêm chế phẩm dược trong quá trình tiến hành thí nghiệm).

\subsection{Xác định thành phần cao chiết}

Từ kết quả khảo sát hoạt tính sinh học của các cao chiết và ảnh hưởng của chúng trên hoạt tính sinh học của doxorubicin đã lựa chọn cao chiết $\mathrm{ZZ}$ là đối tượng nghiên cứu tiếp theo. Thành phần cao chiết $\mathrm{ZZ/EtOH}$ được định tính bằng phương pháp LC/MS tại Viện Hàn Lâm Khoa Học và Công Nghệ Việt Nam (số 1, Mạc Đĩnh Chi, Quận 1). Sau đó kết quả này được tham chiếu với nghiển cứu của tác giả N. J. Yob và các cộng sự [31] tiến hành dự đoán các thành phần hóa học chính của cao chiết. Đồng thời dựa trên kết quả TLC sử dụng hệ dung môi giải ly là hexan: $\mathrm{EtOAc}=1: 1$ tiến hành sắc ký cột với cao chiết $\mathrm{ZZ/EtOH}$ nhằm mục đích tách và thu hồi riêng lẻ các thành phần phục vụ cho việc đánh giá hoạt tính của chúng. Tốc độ giải ly của dung môi là 4-6 giọt/phút, khối lượng mẫu là 2,874 g. Quá trình tách tiếp tục được theo dõi và đánh giá bằng phương pháp TLC.

\section{KẾT QUẢ VÀ BÀN LUẬN Điều kiện chiết tối ưu}

Quá trình chiết được đánh giá trong các khoảng thời gian $1,7,14$ và 30 ngày. Kết quả khảo sát hiệu suất tối ưu được trình bày ở Bảng 1

Bảng 1. Kết quả tối ưu điều kiện thu cao chiết

\begin{tabular}{|c|c|c|c|c|}
\hline Cao chiết & $\begin{array}{c}\text { Thông số } \\
\text { Dung môi chiết tối }\end{array}$ & $\begin{array}{c}\text { Thời gian chiết tối } \\
\text { ưu (ngày) }\end{array}$ & Hiệu suất chiết (\%) & $\begin{array}{c}\text { Hệ dung môi giải ly } \\
\text { (EtOAc:Hexan) } \\
\text { phương pháp TLC }\end{array}$ \\
\hline $\mathrm{ZO}$ & $\mathrm{TCM}$ & 7 & 5.93 & $1: 8$ \\
\hline $\mathrm{ZZ}$ & $\mathrm{EtOH}$ & 7 & 8.00 & $6: 4$ \\
\hline $\mathrm{CC}$ & $\mathrm{TCM}$ & 7 & 4.00 & $1: 6$ \\
\hline $\mathrm{CZ}$ & $\mathrm{TCM}$ & 30 & 5.33 & $1: 9$ \\
\hline $\mathrm{AV}$ & $\mathrm{EtOH}$ & 30 & 1.91 & $1: 2$ \\
\hline
\end{tabular}


Dung môi chiết tối ưu đối với từng nguyên liệu được đánh giá và lựa chọn dựa trên kết quả TLC được khảo sát liên tục trong thời gian $1-30$ ngày. Bảng 1 cho thấy rễ và quả của cây họ gừng thích hợp chiết trong dung môi EtOH và TCM. Khi chiết trong dung môi EtOAc, các cao chiết không hiển thị các vạch chất trên tấm bản mỏng trong suốt thời gian khảo sát. Điều này có thể giải thích là do độ hòa tan kém của các thành phần chất thuộc họ gừng trong dung môi EtOAc.

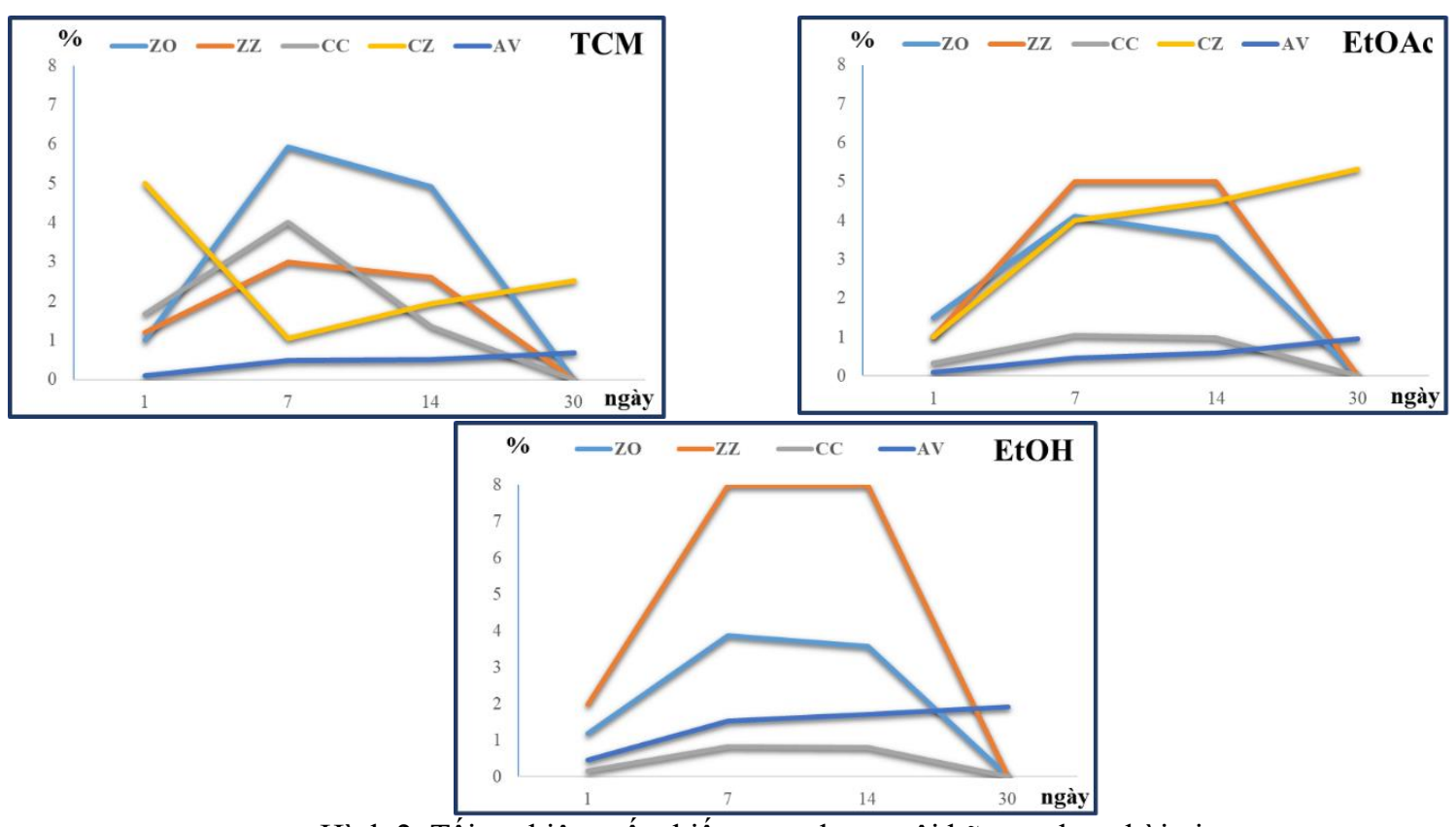

Hình 2. Tối ưu hiệu suất chiết trong dung môi hữu cơ theo thời gian

Kết quả trong Bảng 1 cũng cho thấy hệ dung môi giải ly thích hợp với từng loại cao chiết theo phương pháp TLC nhằm phục vụ cho các bước nghiên cứu tiếp theo.

\section{Hoạt tính sinh học của các cao chiết}

Độc tính và hoạt tính kháng ung thư in vitro của các cao chiết được thể hiện trong Bảng 2 . Để xem xét hoạt tính kháng ung thư và độc tính của các cao chiết lấy mẫu chuẩn là doxorubicin, nghiên cứu sử dụng hai dòng tế bào là tế bào ung thư trực tràng (ruột kết) HCT-116 và nguyên bào sợi da người HSF. Tế bào được nuôi cấy theo tiêu chuẩn quốc tế tại viện nghiên cứu và giáo dục về dược học trường đại học tổng hợp liên bang Kazan, Liên Bang Nga.

Bảng 2. Độc tính và hoạt tính kháng ung thư của các cao chiết

\begin{tabular}{|c|c|c|c|c|c|}
\hline \multirow[t]{2}{*}{ Cao chiết } & $\mathrm{IC}_{50}(\mu \mathrm{g} / \mathrm{mL})$ & $\mathrm{IC}_{25}(\mu \mathrm{g} / \mathrm{mL})$ & $\mathrm{CC}_{50}(\mu \mathrm{g} / \mathrm{mL})$ & $\mathrm{CC}_{25}(\mu \mathrm{g} / \mathrm{mL})$ & \multirow{2}{*}{$\begin{array}{c}\text { Độ chọn lọc }(S I) \\
\mathrm{CC}_{50} / \mathrm{IC}_{50}\end{array}$} \\
\hline & \multicolumn{2}{|c|}{ HSF } & \multicolumn{2}{|c|}{ HCT-116 } & \\
\hline $\mathrm{ZO}$ & $291 \pm 1$ & $213 \pm 2$ & $107 \pm 2$ & $101 \pm 2$ & 0.38 \\
\hline $\mathrm{ZZ}$ & $>1000$ & $40 \pm 1$ & $66 \pm 16$ & $>1000$ & $<0.66$ \\
\hline $\mathrm{CC}$ & $371 \pm 2$ & $321 \pm 2$ & $62 \pm 16$ & $>1000$ & 0.17 \\
\hline $\mathrm{CZ}$ & $50 \pm 9$ & $28 \pm 8$ & $147 \pm 23$ & $107 \pm 27$ & 2.94 \\
\hline $\mathrm{AV}$ & $299 \pm 9$ & $177 \pm 9$ & $115 \pm 33$ & $110 \pm 29$ & 0.38 \\
\hline Doxorubicin & $3917 \pm 4$ & $182 \pm 9$ & $259 \pm 28$ & $78 \pm 16$ & 15,60 \\
\hline
\end{tabular}

* $\mathrm{IC}_{50}$ - The half maximal inhibitory concentration - Nồng độ ức chế tối đa một nửa

* $\mathrm{IC}_{25}$ - The quarter maximal inhibitory concentration - Nồng độ ức chế tối đa một phần tư

* $\mathrm{CC}_{50}$ - The half maximal cytotoxic concentration - Nồng độ gây độc tối đa một nửa

* $\mathrm{CC}_{25}$ - The quarter maximal cytotoxic concentration - Nồng độ gây độc tối đa một phần tư

* SI - Selectivity index - Chỉ số chọn lọc

Kết quả ở Bảng 2 cho thấy cao chiểt CZ thể hiện độc tính trên nguyên bào HSF cao hơn các cao chiết còn lại $\left(\mathrm{IC}_{50}=50 \mu \mathrm{g} / \mathrm{mL} ; \mathrm{IC}_{25}=28 \mu \mathrm{g} / \mathrm{mL}\right)$ trong khi hoạt tính kháng ung thư nằm ở mức trung bình $\left(\mathrm{IC}_{50}=\right.$ $147 \mu \mathrm{g} / \mathrm{mL}$ ). Trong khi đó cao chiết ZZ không gây độc trên nguyên bào HSF ở nồng độ $1000 \mu \mathrm{g} / \mathrm{mL}$, tại nồng độ $40 \mu \mathrm{g} / \mathrm{mL}$ cao chiết $\mathrm{ZZ}$ ức chế $25 \%$ nguyên bào sống sót. Xem xét độ chọn lọc (SI) của các cao 
chiết, tất cả các mẫu được khảo sát đều có độ chọn lọc tốt hơn (thấp hơn) doxorubicin, điều này có thể giải thích do đặc tính tự nhiên của các cao chiết. Trong đó phải kể đến cao chiết $\mathrm{CC}$ có độ chọn lọc thấp nhất (0.17), tại nồng độ $62 \mu \mathrm{g} / \mathrm{mL}$, cao chiết này có thể ức chế sự phát triển của $50 \%$ tế bào ung thư đồng thời ít gây độc trên nguyên bào sợi da người $\left(\mathrm{IC}_{50}=371 \mu \mathrm{g} / \mathrm{mL}\right)$.

Bảng 3 tổng hợp một số nghiên cứu trước đây về hoạt tính kháng ung thư in vitro của các cây thuộc họ Gừng [25-30], có thể thấy giá trị $\mathrm{IC}_{50}$ của các cao chiết được khảo sát nằm trong khoảng $18-385 \mu \mathrm{g} / \mathrm{mL}$ tương đối phù hợp với kết quả nêu ra trong trong nghiên cứu này. Đặc biệt có thể thấy các hoạt tính và thành phần của cao chiết $\mathrm{CC}$ chưa được khảo sát rộng rãi trước đây.

Bảng 3. Hoạt tính sinh học của các cao chiết trong một số nghiên cứu

\begin{tabular}{|c|c|c|c|c|c|c|c|c|}
\hline \multirow{2}{*}{ Mẫu } & \multicolumn{7}{|c|}{$\mathrm{IC}_{50}(\mu \mathrm{g} / \mathrm{mL})$} \\
\cline { 2 - 9 } & HCT-116 & HSF & HepG2 & HL60 & Lym & PC3 & COR-L23 & SNU-1 \\
\hline ZO & $\mathbf{1 0 7} \pm \mathbf{2}$ & $\mathbf{2 9 1} \pm \mathbf{1}$ & - & - & - & $18 \pm 2$ & $122 \pm 2$ & - \\
\hline $\mathrm{ZZ}$ & $\mathbf{6 6} \pm \mathbf{1 6}$ & $\mathbf{> 1 0 0 0}$ & - & $64 \pm 1$ & $385 \pm 40$ & - & - & - \\
\hline $\mathrm{CC}$ & $\mathbf{6 2} \pm \mathbf{1 6}$ & $\mathbf{3 7 1} \pm \mathbf{2}$ & - & - & - & - & - & - \\
\hline $\mathrm{CZ}$ & $\mathbf{1 4 7} \pm \mathbf{2 3}$ & $\mathbf{5 0} \pm \mathbf{9}$ & - & - & - & - & - & $94 \pm 34$ \\
\hline $\mathrm{AV}$ & $\mathbf{1 1 5} \pm \mathbf{3 3}$ & $\mathbf{2 9 9} \pm \mathbf{9}$ & $119 \pm 9$ & - & - & - & - & - \\
\hline
\end{tabular}

* HepG2 - Human hepatocarcinoma - Tế bào ung thư gan

* HL60 - Human myeloid leukemia - Tế bào ung thư máu (bạch cầu tủy)

*Lym - Lymphocytes - Tế bào Lympho

* PC3 - Human Caucasian prostate adenocarcinoma - Tế bào ung thư tuyến tiền liệt

* COR-L23 - Human Caucasian lung large cell carcinoma - Tế bào ung thư biểu mô lớn

* SNU-1 - Gastric carcinoma - ung thư biểu mô dạ dày

Một nghiên cứu đã chỉ ra rằng chiết xuất từ gừng gây nên quá trình chết rụng tế bào ung thư trực tràng HCT-116 và HT-29 [20]. Công thức thảo dược được phát hiện từ y học cổ truyền Trung Quốc có tên C168 (gồm Cinnamomum spp., Zingiber spp., Atractylodes spp., Carthamus spp., Angelica spp., Curcuma spp., Glycyrrhiza spp., và Astragalus spp) đã kích hoạt quá trình chết rụng tế bào ung thư trực tràng HCT-116 thông qua tác động lên sự mất cân bằng oxy hóa và phá hủy các phân tử $\mathrm{ADN}$ [23]. Như vậy có thể thấy việc lựa chọn doxorubicin làm thuốc chuẩn để so sánh đảm bảo tính hợp lý của nghiên cứu vì cơ chế tác dụng của doxorubicin cũng chủ yếu thông qua việc tác dụng lên quá trình nhân bản của các phân tử $\mathrm{ADN}$ trong chu kỳ sinh trưởng của tế bào ung thư kích hoạt sự chết rụng các tế bào này [24].

\section{Ảnh hưởng của cao chiết trên hoạt tính sinh học của doxorubicin}

Để nghiên cứu ảnh hưởng của các cao chiết lên hoạt tính sinh học của Doxorubicin tác giả tiến hành nuôi cấy tế bào trong hỗn hợp doxorubicin-cao chiết được chuẩn bị trong D-MEM, trong đó các cao chiết sử dụng nồng độ $\mathrm{IC}_{25}$ và thay đổi tuyến tính nồng độ của Doxorubicin (từ nồng độ khởi đầu là $1 \mathrm{mg} / \mathrm{mL}$ pha loãng tuyển tính 5 lần tương ứng 5 điểm khảo sát) tiến hành MTT-test cho ra kết quả về hoạt tính và độc tính của doxorubicin được thể hiện ở Bảng 4

Bảng 4. Hoạt tính sinh học của doxorubicin (DOX) khi kết hợp với một số cao chiết thuộc họ Gừng

\begin{tabular}{|c|c|c|c|c|c|}
\hline \multirow{2}{*}{ Mẫu } & $\mathrm{IC}_{50}(\mu \mathrm{g} / \mathrm{mL})$ & $\mathrm{IC}_{25}(\mu \mathrm{g} / \mathrm{mL})$ & $\mathrm{CC}_{50}(\mu \mathrm{g} / \mathrm{mL})$ & $\mathrm{CC}_{25}(\mu \mathrm{g} / \mathrm{mL})$ & Độ chọn lọc $(S I)$ \\
\cline { 2 - 6 } & \multicolumn{2}{|c|}{$\mathrm{HSF}$} & \multicolumn{2}{c|}{$\mathrm{HCT}-116$} & $\mathrm{CC}_{50} / \mathrm{IC}_{50}$ \\
\hline $\mathrm{DOX}+\mathrm{ZZ}$ & $56 \pm 5$ & $27 \pm 9$ & $4 \pm 2$ & $3 \pm 4$ & 0.07 \\
\hline $\mathrm{DOX}+\mathrm{CC}$ & $987 \pm 7$ & $361 \pm 11$ & $106 \pm 22$ & $147 \pm 18$ & 0.11 \\
\hline $\mathrm{DOX}+\mathrm{AV}$ & $633 \pm 19$ & $85 \pm 4$ & $174 \pm 50$ & $136 \pm 48$ & 0.27 \\
\hline DOX & $3917 \pm 4$ & $182 \pm 9$ & $259 \pm 28$ & $78 \pm 16$ & 15,60 \\
\hline
\end{tabular}

Trong các mẫu cao chiết thì mẫu $\mathrm{ZO}$ và $\mathrm{CZ}$ có độ hòa tan kém trong dung môi nuôi cấy ở nồng độ $\mathrm{IC}_{25}$ do đó được xem là mẫu không đạt và không tiến hành khảo sát tiếp. Từ Bảng 4 có thể thấy cả 3 cao chiết $Z Z$, $\mathrm{CC}$ và $\mathrm{AV}$ đều có tác động tích cực trên hoạt tính sinh học của doxorubicin, cụ thể $\mathrm{ZZ}$ tăng hoạt tính kháng ung thư in vitro của doxorubicin lên 65 lần, $\mathrm{CC} 2.5$ lần và $\mathrm{AV} 1.5$ lần. Bên cạnh đó cả ba cao chiết này đồng thời tăng độc tính của doxorubicin trên nguyên bào sợi da người (ZZ 70 lần, CC 4 lần và $\mathrm{AV} 6.2$ lần) (Hình 3) 

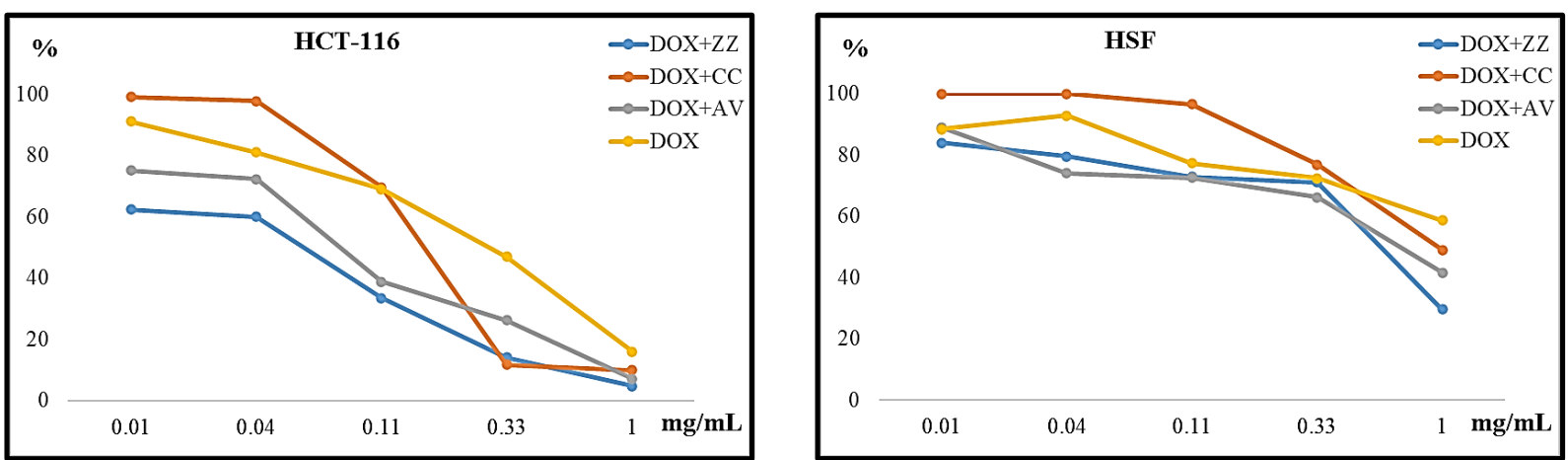

Hình 3. Ảnh hưởng của các cao chiết họ Gừng trên hoạt tính sinh học của doxorubicin

Từ kết quả ở Bảng 4 cho thấy khi so sánh độ chọn lọc của các mẫu khảo sát có thể thấy cả ba cao chiết đều giúp tăng đáng kể độ chọn lọc của doxorubicin (ZZ 223 lần, CC 142 lần, AV 58 lần). Ngoài ra từ đồ thị Hình 3 có thể thấy khi sử dụng đồng thời hỗn hợp doxorubicin-cao chiết thì doxorubicin tại nồng độ $0.33 \mathrm{mg} / \mathrm{mL}$ đã thể hiện hoạt tính kháng ung thư in vitro cao hơn rất nhiều (\% tế bào sống sót thấp) so với việc chỉ sử dụng doxorubicin (đối với tế bào $\mathrm{HCT}-116$ ). Trong đó cao chiết $\mathrm{ZZ}$ được đánh giá là có ảnh hưởng tốt nhất trên hoạt tính sinh học của doxorubicin được chọn làm đối tượng nghiên cứu trong các bước tiếp theo.

Trong một nghiên cứu của minh, Nahla E. El Ashmawy và các cộng sự đã cho thấy liệu pháp sử dụng đồng thời chiết xuất gừng và doxorubicin cho hiệu quả kháng ung thư biểu mô ở chuột cao hơn khi chỉ sử dụng riêng lẻ một trong hai loại dược phẩm này [21]. Bên cạnh đó việc tạo ra một công thức tương thích sinh học giữa 6-gingerol, doxorubicin và alginate/hydroxyapatite đã cho thấy tác dụng chống tăng sinh trên hai dòng tế bào ung thư biểu mô vú MCF-7 và ung thư biểu mô tế bào gan HEpG2 [22]. Điều này đặt ra mục tiêu tiếp theo cho nghiên cứu là tìm ra thành phần hóa học chính của cao chiết $\mathrm{ZZ}$ cũng như khảo sát cơ chế ức chế ung thư của hỗn hợp doxorubicin-ZZ/EtOH.

\section{Phổ LC/MS cao chiết ZZ}

Thành phần có trong cao chiết ZZ thu hoạch tại huyện Phú Riềng, tỉnh Bình Phước được xác định bằng phương pháp LC/MS. Mẫu cao chiết được phân tích trên Hệ thống sắc ký Dionex của hãng Thermo Scientific (Mỹ ) sử dụng phần mềm Chromeleon phiên bản 7.2.4.8179 với hệ thống UV-VIS-3 với bước sóng $280 \mathrm{~nm}$ thu được kết quả biểu diễn trong Hình 4.

Dựa vào kết quả LC/MS cao chiết $\mathrm{ZZ}$ thu hoạch tại tỉnh Bình Phước có chứa một số thành phần đặc trưng được dự đoán là cineol $\left(\mathrm{Mw}=204.357 \mathrm{~g} \cdot \mathrm{mol}^{-1}\right)$ thuộc peak 9; kaempferol $\left(\mathrm{Mw}=286.236 \mathrm{~g} \cdot \mathrm{mol}^{-1}\right)$ thuộc peak 11; humulene, zingiberene, cadinene, $\beta$-caryophyllene $\left(\mathrm{Mw}=204.357{\mathrm{~g} . \mathrm{mol}^{-1}}^{-1}\right.$, zerumbone $\left(\mathrm{Mw}=218.330 \mathrm{~g} \cdot \mathrm{mol}^{-1}\right)$ thuộc peak 18; cynaropicrin $\left(\mathrm{Mw}=346.379 \mathrm{~g} \cdot \mathrm{mol}^{-1}\right)$ thuộc peak 15; diferuloylmethane $\left(\mathrm{Mw}=368.385 \mathrm{~g} \cdot \mathrm{mol}^{-1}\right)$, quercitrin hydrate $\left(\mathrm{Mw}=466.386 \mathrm{~g} \cdot \mathrm{mol}^{-1}\right)$, 4-nitrophenyl $\alpha$-Lrhamnopyranoside $\left(\mathrm{Mw}=285.254 \mathrm{~g} \cdot \mathrm{mol}^{-1}\right)$ thuộc peak 21 ; 4-nitrophenyl $\alpha$-L-rhamnopyranoside $(\mathrm{Mw}=$ $\left.285.254 \mathrm{~g} \cdot \mathrm{mol}^{-1}\right)$, dimethoxycurcumin $\left(\mathrm{Mw}=396.398 \mathrm{~g} \cdot \mathrm{mol}^{-1}\right)$, vitexin $\left(\mathrm{Mw}=432.381 \mathrm{~g} \cdot \mathrm{mol}^{-1}\right)$ thuộc peak 24 phù hợp với các nghiên cứu trước đây [31]. Một số monoterpene có khối lượng phân tử thấp hơn thường gặp trong tinh dầu gừng như pinene, camphene, limonene không được phát hiện trong kết quả LC/MS nhận được. Như vậy có thể thấy hoạt tính sinh học cao của cao chiết $\mathrm{ZZ}$ có thể liên quan tới sự có mặt của các thành phần kề trên. 


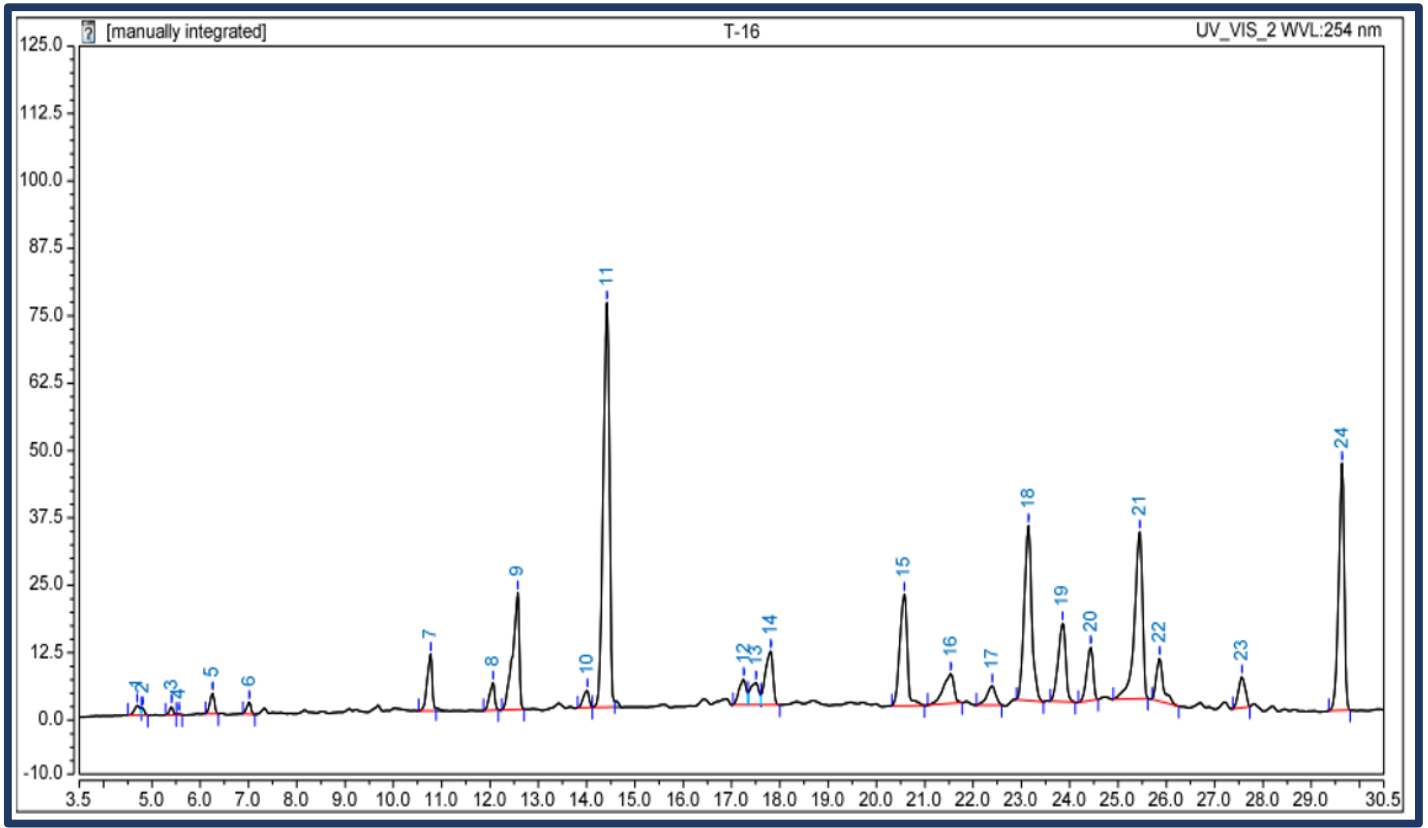

Hình 4. Phổ LC/MS cao chiết ZZ

Kết quả định tính bằng phương pháp LC/MS xác định có 24 hợp chất chính trong cao chiết $\mathrm{ZZ}$. Trong đó một số peak được xác định với hàm lượng \% cao bao gồm peak 9 (6.73\%), peak $11(23.29 \%)$, peak 15 (6.42\%), peak 18 (10.08\%), peak 21 (9.61\%), peak 24 (14.22\%) và các peak khác (Bảng 5).

Bảng 5. Hàm lượng \% các peak trên phổ LC/MS cao chiết ZZ

\begin{tabular}{|c|c|c|c|c|c|}
\hline STT & $\begin{array}{l}\text { Thời gian lưu } \\
\text { mẫu (phút) }\end{array}$ & $\begin{array}{c}\text { Diện tích } \\
\text { (mAU*phút) }\end{array}$ & $\begin{array}{c}\text { Chiều cao } \\
\text { (mAU) }\end{array}$ & $\begin{array}{c}\text { Diện tích tương } \\
\text { đối }(\%)\end{array}$ & $\begin{array}{c}\text { Chiều cao tương } \\
\text { đối }(\%)\end{array}$ \\
\hline 1 & 4.700 & 0.248 & 1.783 & 0.48 & 0.55 \\
\hline 2 & 4.807 & 0.098 & 1.276 & 0.19 & 0.40 \\
\hline 3 & 5.407 & 0.139 & 1.630 & 0.27 & 0.51 \\
\hline 4 & 5.560 & 0.014 & 0.180 & 0.03 & 0.06 \\
\hline 5 & 6.257 & 0.402 & 3.735 & 0.78 & 1.16 \\
\hline 6 & 7.017 & 0.225 & 2.203 & 0.43 & 0.68 \\
\hline 7 & 10.770 & 1.258 & 10.523 & 2.43 & 3.27 \\
\hline 8 & 12.063 & 0.621 & 5.086 & 1.20 & 1.58 \\
\hline 9 & 12.573 & 3.440 & 21.653 & 6.63 & 6.73 \\
\hline 10 & 14.013 & 0.436 & 3.162 & 0.84 & 0.98 \\
\hline 11 & 14.420 & 11.264 & 74.991 & 21.72 & 23.30 \\
\hline 12 & 17.250 & 0.798 & 4.727 & 1.54 & 1.47 \\
\hline 13 & 17.507 & 0.857 & 4.083 & 1.65 & 1.27 \\
\hline 14 & 17.810 & 1.826 & 9.950 & 3.52 & 3.09 \\
\hline 15 & 20.580 & 3.702 & 20.656 & 7.14 & 6.42 \\
\hline 16 & 21.533 & 1.419 & 5.479 & 2.74 & 1.70 \\
\hline 17 & 22.397 & 0.748 & 3.571 & 1.44 & 1.11 \\
\hline 18 & 23.147 & 5.848 & 32.438 & 11.28 & 10.08 \\
\hline 19 & 23.857 & 2.626 & 14.512 & 5.06 & 4.51 \\
\hline 20 & 24.433 & 1.502 & 9.851 & 2.90 & 3.06 \\
\hline 21 & 25.447 & 5.954 & 30.921 & 11.48 & 9.61 \\
\hline 22 & 25.880 & 1.358 & 8.007 & 2.62 & 2.49 \\
\hline 23 & 27.563 & 0.931 & 5.738 & 1.80 & 1.78 \\
\hline 24 & 29.637 & 6.151 & 45.763 & 11.86 & 14.22 \\
\hline \multicolumn{2}{|r|}{ Tổng } & 51.865 & 321.918 & 100.00 & 100.00 \\
\hline
\end{tabular}

*AU: Absorbance Unit - đơn vị hấp thụ 
Từ kết quả phân tích định tính LC/MS ta nhận thấy rằng ZZ thu hoạch trên địa bàn tỉnh Bình Phước chứa một số hợp chất có nhóm chức chalcone trong phân tử như diferuloylmethane, dimethoxycurcumin. Ngoài các hợp chất được phát hiện trong phổ LC/MS của cao chiết này hầu hết có chứa liên kết đôi hoặc liên hiệp các liên kết đôi với nhiều nhóm methyl- trong phân tử, điều này phù hợp với nghiên cứu của Korytnyk và các đồng nghiệp của ông trong nghiên cứu về hoạt tính kháng ung thư các dẫn xuất vinyl của pyridoxine $[32,33]$ các nghiên cứu này khẳng định hoạt tính kháng ung thư cao của các dẫn xuất vinyl, tuy nhiên độc tính của chúng cũng khá cao.

\section{Sắc ký cột}

Sau khi tiến hành khảo sát hoạt tính sinh học và phân tích định tính thành phần hóa học của cao chiết $\mathrm{ZZ/EtOH}$, tác giả tiến hành phân lập các thành phần này bằng phương pháp sắc ký cột mục đích tách riêng và xác định cấu trúc phân tử của các hợp chất tách được. Tiến hành chạy sắc ký sử dụng hệ dung môi giải ly hexan:EtOAc = 1:1, tốc độ giải ly của dung môi là 4-6 giọt/phút, khối lượng mẫu ban đầu là $2,874 \mathrm{~g}$. Theo dõi quá trình tách bằng sắc ký bản mỏng (TLC). Kết quả phân lập được 26 mẫu trong đó có 12 hỗn hợp các vạch chất và 14 hợp chất riêng biệt cho hiệu suất 1,04-6,30\%. Các thành phần này hiện trong giai đoạn nghiên cứu hoạt tính sinh học in vitro sau đó tiến hành định tính/ định lượng bằng phương pháp phổ cộng hưởng từ NMR cho kết quả chính xác hơn.

\section{KẾT LUẬn}

Họ Gừng được xem là một họ cây lâu đời và phổ biến ở Việt Nam với nhiều công dụng khác nhau. Nhiều nghiên cứu khoa học đã chỉ ra các tính chất dược lý vượt trội của một số hợp chất tồn tại trong các cây họ Gừng. Các kết quả nghiên cứu trên cũng cho thấy cao chiết thu được từ rễ và quả của một số cây họ gừng bao gồm Zingiber officinale, Zingber zerumbet, Curcuma clovisii, Curcuma zedoaria và Amomum villosum thể hiện hoạt tính kháng tế bào ung thư trực tràng HCT-116 cao và đồng thời cho chỉ số chọn lọc tốt hơn doxorubicin. Ngoài ra các cao chiết này cũng có ảnh hưởng tích cực trên hoạt tính sinh học và chỉ số chọn lọc của doxorubicin. Kết quả này có ý nghĩa khoa học và thực tiễn cao. Ngoài ra bằng cách so sánh với tài liệu nghiên cứu trước đây tác giả dự đoán được khoảng 24 hợp chất chính có trong thành phần cao chiết $\mathrm{ZZ/EtOH}$. Bằng cách phân lập các thành phân riêng biệt trong cao chiết sẽ giúp xác định chính xác hoạt tính của từng thành phần từ đó định hướng cho ra đời các sản phẩm thực phẩm chức năng hoặc dược phẩm hỗ trợ trong việc ngăn ngừa và điều trị ung thư theo phương pháp kết hợp giữa tây y (doxorubicin) và đông y giảm thiểu các tác dụng phụ và độc tính không đáng có của tây y trên cơ thể bệnh nhân.

\section{LỜI CẢM ƠN}

Các tác giả chân thành cảm ơn sự hỗ trợ tài chính của trường Đại học công nghiệp Thành phố Hồ Chí Minh.

\section{TÀI LIỆU THAM KHẢO}

[1] A. Pugazhendhi, T. Edison, B. K. Velmurugan, J. A. Jacob, and I. Karuppusamy, Toxicity of Doxorubicin (Dox) to different experimental organ systems, Life Sci, vol. 200, pp. 26-30, 2018.

[2] H. Wu et al., Synergistic Cisplatin/Doxorubicin Combination Chemotherapy for Multidrug-Resistant Cancer via Polymeric Nanogels Targeting Delivery, ACS Appl Mater Interfaces, vol. 9, no. 11, pp. 9426-9436, 2017.

[3] L. Liao et al., A convergent synthetic platform for single-nanoparticle combination cancer therapy: ratiometric loading and controlled release of cisplatin, doxorubicin, and camptothecin, J Am Chem Soc, vol. 136, no. 16, pp. 5896-5899, 2014.

[4] C. F. Thorn et al., Doxorubicin pathways: pharmacodynamics and adverse effects, Pharmacogenet Genomics, vol. 21, no. 7, pp. 440-446, 2011.

[5] R. S. Pavelyev et al., Synthesis and in vitro antitumor activity of novel alkenyl derivatives of pyridoxine, bioisosteric analogs of feruloyl methane, Bioorg Med Chem, vol. 26, no. 22, pp. 5824-5837, 2018.

[6] N. Phuong Hanh and N. Quoc Binh, Distribution of Alpinia (Zingiberaceae) and their use pattern in Vietnam, Journal of Biodiversity \& Endangered Species, vol. 02, no. 02, 2014. 
[7] I. N. Chen, C. C. Chang, C. C. Ng, C. Y. Wang, Y. T. Shyu, and T. L. Chang, Antioxidant and antimicrobial activity of Zingiberaceae plants in Taiwan, Plant Foods Hum Nutr, vol. 63, no. 1, pp. 15-20, 2008.

[8] D. Suthisut, P. G. Fields, and A. Chandrapatya, Contact toxicity, feeding reduction, and repellency of essential oils from three plants from the ginger family (Zingiberaceae) and their major components against Sitophilus zeamais and Tribolium castaneum, J Econ Entomol, vol. 104, no. 4, pp. 1445-1454, 2011.

[9] I. Batubara, Y. Kartika, and L. K. Darusman, Relationship between Zingiberaceae Leaves Compounds and its Tyrosinase Activity, Biosaintifika: Journal of Biology \& Biology Education, vol. 8, no. 3, 2016.

[10] I. Batubara, W. T. Wahyuni, and M. Susanta, Antibacterial activity of zingiberaceae leaves Essential oils against streptococcus mutans And teeth-biofilm degradation, International Journal of Pharma and Bio Sciences, vol. 7, no. 4, 2016.

[11] S. Wang et al., Antidiarrheal effect of Alpinia oxyphylla Miq. (Zingiberaceae) in experimental mice and its possible mechanism of action, J Ethnopharmacol, vol. 168, pp. 182-190, 2015.

[12] S. Tewtrakul and S. Subhadhirasakul, Anti-allergic activity of some selected plants in the Zingiberaceae family, J Ethnopharmacol, vol. 109, no. 3, pp. 535-538, 2007.

[13] M. Govindarajan, M. Rajeswary, S. Arivoli, S. Tennyson, and G. Benelli, Larvicidal and repellent potential of Zingiber nimmonii (J. Graham) Dalzell (Zingiberaceae) essential oil: an eco-friendly tool against malaria, dengue, and lymphatic filariasis mosquito vectors?, Parasitol Res, vol. 115, no. 5, pp. 1807-1816, 2016.

[14] J. Wang, W. Ke, R. Bao, X. Hu, and F. Chen, Beneficial effects of ginger Zingiber officinale Roscoe on obesity and metabolic syndrome: a review, Ann N Y Acad Sci, vol. 1398, no. 1, pp. 83-98, 2017.

[15] M. Chetia, B. R. Giri, A. Swargiary, B. Ronghang, and B. Roy, Amomum maximum Roxb (Zingiberaceae), a Medicinal Plant of Tripura, India: A Natural Anthelmintic?, Journal of Advanced Microscopy Research, vol. 9 , no. 2, pp. 148-153, 2014.

[16] B. Roy, A. Swargiary, and B. Ranjan Giri, Alpinia Nigra (Family Zingiberaceae): An Anthelmintic Medicinal Plant of North-East India, Advances in Life Sciences, vol. 2, no. 3, pp. 39-51, 2012.

[17] A. S. Nagaraja et al., Sustained adrenergic signaling leads to increased metastasis in ovarian cancer via increased PGE2 synthesis, Oncogene, vol. 35, no. 18, pp. 2390-2397, 2016.

[18] B. Fuller, Role of PGE-2 and Other Inflammatory Mediators in Skin Aging and Their Inhibition by Topical Natural Anti-Inflammatories, Cosmetics, vol. 6, no. 1, 2019.

[19] Y. Ding et al., Chromatin remodeling ATPase BRG1 and PTEN are synthetic lethal in prostate cancer, J Clin Invest, vol. 129, no. 2, pp. 759-773, 2011.

[20] Shailah Abdullah, Siti Amalina Zainal Abidin, Noor Azian Murad, Suzana Makpol, Wan Zurinah Wan Ngah, and Yasmin Anum Mohd Yusof, Ginger extract (Zingiber officinale) triggers apoptosis and G0/G1 cells arrest in HCT 116 and HT 29 colon cancer cell lines. African Journal of Biochemistry Research, vol. 4, no. 4, pp. 134$142,2010$.

[21] Nahla E. El-Ashmawy, Naglaa F. Khedr, Hoda A. El-Bahrawy, Hend E. Abo Mansour, Ginger extract adjuvant to doxorubicin in mammary carcinoma: study of some molecular mechanisms, Eur J Nutr, vol. 57, no. 3, pp. 981 989, 2017.

[22] Manatunga, D. C., de Silva, R. M., de Silva, K. M. N., Wijeratne, D. T., Malavige, G. N., Williams, G., Fabrication of 6-gingerol, doxorubicin and alginate hydroxyapatite into a bio-compatible formulation: enhanced 
anti-proliferative effect on breast and liver cancer cells, Chemistry Central Journal, vol. 12, no. 1, pp. 119-131, 2018.

[23] Leong, L. M., Chan, K. M., Hamid, A., Latip, J., Rajab, N. F., Herbal Formulation C168 Attenuates Proliferation and Induces Apoptosis in HCT 116 Human Colorectal Carcinoma Cells: Role of Oxidative Stress and DNA Damage, Evidence-Based Complementary and Alternative Medicine, pp. 1-12, 2016.

[24] Cristina Pérez-Arnaiz, Natalia Busto, José M. Leal, and Begoña García, New Insights into the Mechanism of the DNA/Doxorubicin Interaction, J. Phys. Chem. B, vol. 118, no. 5, pp. 1288-1295, 2014.

[25] Sasipawan Machana et al., Cytotoxic and apoptotic effects of six herbal plants against the human hepatocarcinoma (HepG2) cell line, Chinese Medicine, vol. 6, no. 39, pp. 1-8, 2019.

[26] Norfazlina Mohd Nawi, Mohd Nazip Suratman, In vitro cytotoxicity effects of single and combination Nigella sativa and Zingiber zerumbet extracts on human myeloid leukemia (HL60) cells and its mode of cell death, Journal of Applied Pharmaceutical Science, vol. 4, no. 05, pp. 051-055, 2014.

[27] Anish Nag, Maumita Bandyopadhyay, Anita Mukherjee, Antioxidant Activities and Cytotoxicity of Zingiber zerumbet (L.) Smith Rhizome, Journalof Pharmacognosy and Phytochemistry, vol. 2, no. 3, pp. 102-108, 2013.

[28] Lobo, R., Prabhu, K. S., Shirwaikar, A., Shirwaikar, A., Curcuma zedoaria Rosc. (white turmeric): a review of its chemical, pharmacological and ethnomedicinal properties, J Pharm Pharmacol, vol. 61, no. 1, pp. 13-21, 2009.

[29] Athima Saetung et al., Cytotoxic activity of Thai medicinal plants for cancer treatment, Songklanakarin J. Sci. Technol, vol. 27, no. 2, pp. 469-478, 2005.

[30] Kirana, Chandra, Record, Ian R., McIntosh, Graeme H., Jones, Graham P., Screening for Antitumor Activity of 11 Species of Indonesian Zingiberaceae Using Human MCF-7 and HT-29 Cancer Cells, Pharmaceutical Biology, vol. 41, no. 4, pp. 271-276, 2003.

[31] N. J. Yob, S. M. Jofrry, M. M. Affandi, L. K. Teh, M. Z. Salleh, and Z. A. Zakaria, Zingiber zerumbet (L.) Smith: A Review of Its Ethnomedicinal, Chemical, and Pharmacological Uses, Evid Based Complement Alternat Med, vol. 2011, p. 543216, 2011.

[32] N. A. W. Korytnyk, B. Lachmann, P.G.G. Potti, Substrate specificity of pyridoxine dehydrogenase from yeast, and the synthesis and biological activities of 5-vinyl and 5-ethynyl analogs of pyridoxo1, J. Med. Chem. , vol. 15, no. $12,1972$.

[33] S. C. S. W. Korytnyk, N.Angelino, P.G. Potti, B. Paul, General method for modifying the 2-methyl group of pyridoxol. synthesis and biological activity of 2-vinyl- and 2-ethynylpyridoxols and related compounds, J. Paul, Med. Chem. , vol. 16, pp. 1096-1101, 1973.

Ngày nhận bài: 30/06/2020

Ngày chấp nhận đăng: 24/12/2020 